\title{
Relations Between the Product- and Sum-connectivity Indices
}

\author{
Bo Zhou ${ }^{\mathrm{a}, *}$ and Nenad Trinajstićc \\ ${ }^{a}$ Department of Mathematics, South China Normal University, Guangzhou 510631, China \\ ${ }^{\mathrm{b}}$ The Rugjer Bošković Institute, P. O. Box 180, HR-10002 Zagreb, Croatia
}

RECEIVED JANUARY 17, 2012; ACCEPTED SEPTEMBER 28, 2012

Abstract. Several relations between the product- and sum-connectivity indices are established. (doi: $10.5562 /$ cca2052)

Keywords: Randić connectivity index, product-connectivity index, sum-connectivity index, vertex degree

\section{INTRODUCTION}

Let $G$ be a simple graph with vertex-set $V(G)$ and edgeset $E(G) .{ }^{1}$ For $v \in V(G), \Gamma(v)$ denotes the set of its (first) neighbors in $G$ and the degree of $v$ is $d_{v}=|\Gamma(v)|$. The product-connectivity index or the Randić connectivity index $R=R(G)$ of $G$ is defined as ${ }^{2}$

$$
R(G)=\sum_{u v \in E(G)} \frac{1}{\sqrt{d_{u} d_{v}}}
$$

The product-connectivity index is used successfully in QSPR and QSAR modeling ${ }^{3-10}$ and its mathematical properties have also been studied extensively, as summarized in two monographs. ${ }^{11,12}$

The sum-connectivity index $\chi=\chi(G)$ of $G$ is defined as ${ }^{13}$

$$
\chi(G)=\sum_{u v \in E(G)} \frac{1}{\sqrt{d_{u}+d_{v}}} .
$$

The use of sum-connectivity index in QSPR and QSAR modeling has been investigated. ${ }^{14-16}$ As already pointed by Balaban et al., ${ }^{17}$ the sum-connectivity index may be considered as a proper topological index ${ }^{1}$ (molecular descriptor ${ }^{6,7}$ ). Mathematical properties of the sum-connectivity index have also been established. ${ }^{18-21}$ Related to the sum-connectivity index, sum-connectivity matrix and sum-connectivity energy have been proposed. ${ }^{22}$

In this note, we establish several relations between the product- and the sum-connectivity indices.

\section{RELATIONS BETWEEN THE PRODUCT- AND SUM-CONNECTIVITY INDICES}

Let $G$ be a graph. If $G$ has no pendant vertices (vertices of degree one), then $\chi(G) \geq R(G)$ with equality if and only if all non-isolated vertices have degree two. ${ }^{13}$ Obviously, $d_{u}+d_{v} \leq 2 d_{u} d_{v}$ with equality if and only if $d_{u}=d_{v}=1$. Thus

$$
\chi(G)=\sum_{u v \in E(G)} \frac{1}{\sqrt{d_{u}+d_{v}}} \geq \sum_{u v \in E(G)} \frac{1}{\sqrt{2 d_{u} d_{v}}}=\frac{1}{\sqrt{2}} R(G)
$$

with equality if and only if all non-isolated vertices have degree one. If $d_{u} \geq d_{v} \geq 1$ and $d_{u} \geq 2$, then $d_{u}+d_{v} \leq \frac{3}{2} d_{u} d_{v}$ with equality if and only if $d_{u}=2$ and $d_{v}=1$. Thus, if $G$ has no components on two vertices, then

$$
\chi(G)=\sum_{u v E(G)} \frac{1}{\sqrt{d_{u}+d_{v}}} \geq \sum_{u v \in E(G)} \frac{1}{\sqrt{\frac{3}{2} d_{u} d_{v}}}=\sqrt{\frac{2}{3}} R(G)
$$

with equality if and only if all non-trivial components of $G$ are paths on three vertices. Thus we have

Proposition 1. Let $G$ be a graph. Then $\chi(G) \geq$ $\frac{1}{\sqrt{2}} R(G)$ with equality if and only if all non-isolated vertices have degree one. Moreover, if $G$ has no components on two vertices, then $\chi(G) \geq \sqrt{\frac{2}{3}} R(G)$ with equality

\footnotetext{
* Author to whom correspondence should be addressed. (E-mail: zhoubo@scnu.edu.cn)
} 
if and only if all non-trivial components of $G$ are paths on three vertices, and if no pendant vertices, then $\chi(G) \geq R(G)$ with equality if and only if all non-isolated vertices have degree two.

Let $G$ be a graph with $n$ vertices and $m$ edges. By Caychy-Schwarz inequality,

$$
\chi(G)=\sum_{u v \in E(G)} \frac{1}{\sqrt{d_{u}+d_{v}}} \leq \sqrt{m \sum_{u v \in E(G)} \frac{1}{d_{u}+d_{v}}}
$$

with equality if and only if the sum of degrees of the two end vertices is a constant for all edges. On the other hand, by the arithmetic-geometric mean inequality,

$$
\sum_{u v \in E(G)} \frac{1}{d_{u}+d_{v}} \leq \sum_{u v \in E(G)} \frac{1}{2 \sqrt{d_{u} d_{v}}}=\frac{R(G)}{2}
$$

with equality if and only if every component is regular. Combining the above two inequalities, we have

Proposition 2. Let $G$ be a graph with $m$ edges. Then

$$
\chi(G) \leq \sqrt{\frac{m R(G)}{2}}
$$

with equality if and only if $G$ is regular.

From Theorem 1, upper bounds for the productconnectivity index will result in upper bounds for the sum-connectivity index. We give several such examples.

Recall that $R(G) \leq \frac{n}{2}$ with equality if and only if every component is nontrivial and regular. ${ }^{11}$ Thus we have Corollary $3 .{ }^{23}$ Let $G$ be a graph with $n$ vertices and $m$ edges. Then

$$
\chi(G) \leq \frac{\sqrt{n m}}{2}
$$

with equality if and only if $G$ is regular.

By Corollary 3, among $n$-vertex graphs the complete graph is the unique graph with maximum sumconnectivity index, equal to $\frac{n \sqrt{n-1}}{2 \sqrt{2}},{ }^{13}$ and among $n$-vertex bipartite graphs the complete bipartite graph with one partite set with $\left\lfloor\frac{n}{2}\right\rfloor$ vertices is the unique graph with maximum sum-connectivity index, equal to $\sqrt{\frac{n}{4}\left\lfloor\frac{n^{2}}{4}\right\rfloor}$.
Let $\bar{G}$ be the complement of the graph $G$. Let $\bar{m}=|E(\bar{G})|$. From Corollary 3, we have

$$
\begin{aligned}
\chi(G)+\chi(\bar{G}) & \leq \frac{\sqrt{n}}{2}(\sqrt{m}+\sqrt{\bar{m}}) \\
& \leq \frac{\sqrt{n}}{2} \cdot \sqrt{2(m+\bar{m})} \\
& =\frac{\sqrt{n}}{2} \cdot \sqrt{n(n-1)} \\
& =\frac{n \sqrt{n-1}}{2}
\end{aligned}
$$

with equalities if and only if $G$ and $\bar{G}$ are regular and $m=\bar{m}$, i.e., $G$ is regular and $2 m=\frac{n(n-1)}{2}$, i.e., $G$ is regular of degree $\frac{n-1}{2}$. Thus we have

Corollary 4. Let $G$ be a graph with $n$ vertices. Then

$$
\chi(G)+\chi(\bar{G}) \leq \frac{n \sqrt{n-1}}{2}
$$

with equality if and only if $G$ is regular of degree $\frac{n-1}{2}$.

Let $G$ be a graph with $n$ vertices. Recall that $\chi(G)+\chi(\bar{G}) \geq \frac{n \sqrt{n-1}}{2 \sqrt{2}}$ with equality if and only if $G$ or $\bar{G}$ is the complete graph. ${ }^{13}$

Let $G$ be a graph with no isolated vertices and clique number $\omega$. A particular result in Reference 24 says $R \leq \frac{\omega-1}{2 \omega}\left(\sum_{u \in V(G)} \frac{1}{\sqrt{d_{u}}}\right)^{2}$ with equality if and only if $G$ is regular complete $\omega$-partite graph. Thus we have

Corollary 5. Let $G$ be a graph with no isolated vertices, $m$ edges and clique number $\omega$. Then

$$
\chi(G) \leq \frac{1}{2} \sqrt{\frac{\omega-1}{\omega} m} \sum_{u \in V(G)} \frac{1}{\sqrt{d_{u}}}
$$

with equality if and only if $G$ is regular complete $\omega$ partite graph.

Acknowledgements. This work was supported by the Guangdong Provincial Science Foundation of China (Grant No. S2011010005539) and the Ministry of Science, Education and Sports of Croatia (Grant No. 098-1770495-2919). 


\section{REFERENCES}

1. N. Trinajstić, Chemical Graph Theory, 2nd revised ed., CRC Press, Boca Raton,. FL, 1992.

2. M. Randić, J. Am. Chem. Soc. 97 (1975) 6609-6615.

3. L. B. Kier and L. H. Hall, Molecular Connectivity in Chemistry and Drug Research, Academic Press, New York, 1976.

4. L. B. Kier and L. H. Hall, Molecular Connectivity in StructureActivity Analysis, Research Studies Press/Wiley, Letchworth/New York, 1986.

5. J. Devillers and A. T. Balaban (eds.), Topological Indices and Related Descriptors in QSAR and QSPR, Gordon and Breach, 1999.

6. R. Todeschini and V. Consonni, Handbook of Molecular Descriptors, Wiley-VCH, Weinheim, 2000.

7. M. V. Diudea (Ed.), QSPR/QSAR Studies by Molecular Descriptors, Nova, Hungtin, N.Y., 2001.

8. L. Pogliani, Chem. Rev. 100 (2000) 3827-3858.

9. M. Randić, J. Mol. Graph. Model. 20 (2001) 19-35.

10. R. García-Domenech, J. Gálvez, J. V. de Julián-Ortiz, and L. Pogliani, Chem. Rev. 108 (2008) 1127-1169.

11. X. Li and I. Gutman, Mathematical Aspects of Randić-Type Molecular Structure Descriptors, University of Kragujevac, Kragujevac, 2006.
12. I. Gutman and B. Furtula (Eds.), Recent Results in the Theory of Randic Index, University of Kragujevac, Kragujevac, 2008.

13. B. Zhou and N. Trinajstić, J. Math. Chem. 46 (2009) 1252-1270.

14. B. Lučić, N. Trinajstić, and B. Zhou, Chem. Phys. Lett. 475 (2009) 146-148.

15. B. Lučić, S. Nikolić, N. Trinajstić, B. Zhou, and S. Ivaniš Turk, in: I. Gutman, and B. Furtula (Eds.), Novel Molecular Structure Descriptors - Theory and Applications I, University of Kragujevac, Kragujevac, 2010, pp. 101-136.

16. D. Vukičević and N. Trinajstić, Croat. Chem. Acta 83 (2010) 349-351.

17. A. T. Balaban, P. V. Khadikar, and S. Aziz, Iranian J. Math. Chem. 1 (2010) 43-67.

18. Z. Du, B. Zhou, and N. Trinajstić, J. Math. Chem. 47 (2010) $842-855$.

19. R. Xing, B. Zhou, and N. Trinajstić, J. Math. Chem. 48 (2010) 583-591.

20. S. Wang, B. Zhou, N. Trinajstić, Filomat 25 (no. 3) (2011) 29-42.

21. Z. Du and B. Zhou, Bull. Malays. Math. Sci. Soc. 35 (2012) 101-117.

22. B. Zhou and N. Trinajstić, Acta Chim. Slov. 57 (2010) 513-517.

23. B. Horoldagva and I. Gutman, MATCH Commun. Math. Comput. Chem. 65 (2011) 723-730.

24. B. Zhou and D. Vukičević, MATCH Commun. Math. Comput Chem. 62 (2009) 189-196. 